\title{
Dynamic Spectrum Sharing Models for Cognitive Radio Aided Ad Hoc Networks and Their Performance Analysis
}

\author{
Gan Liu ${ }^{\dagger \dagger}, \mathrm{Xu}$ Zhu* and Lajos Hanzo \\ $\dagger$ School of Electronics and Computer Science, University of Southampton, Southampton, SO17 1BJ, UK \\ $\ddagger$ Wuhan National Laboratory for Optoelectronics, Department of Electronics and Information Engineering, Huazhong University \\ of Science and Technology, Wuhan, 430074, China. \\ * Department of Electrical Engineering and Electronics, University of Liverpool, Liverpool, L69 3GJ, UK \\ Emails: g111v@ecs.soton.ac.uk; xuzhu@liv.ac.uk; lh@ecs.soton.ac.uk
}

\begin{abstract}
In this paper, two dynamic spectrum sharing models are proposed, namely a Markov-chain model and a queue-based model in order, to evaluate the performance of CRAHNs, where the shared Primary Channels (PCs) are complemented by unshared Secondary Channels (SCs). The new contribution of this paper is as follows. Firstly, our Markov-chain model is extendable to any practical number of PCs and SCs and remains accurate for any practical Primary User (PU) and Secondary User (SU) tele-traffic intensity. As a benefit, our technique accurately estimates the maximum number of Secondary Users Per Second (NSUPS) supported by a system, both analytically and by simulations. In addition to our Markov-chain model, we also conceive queue-based models, which generally impose a lower modeling complexity than that of Markov-chain models, although at the cost of being more inaccurate. Our numerical results confirm the reduced evaluation complexity and improved accuracy of the proposed models and analysis.
\end{abstract}

Keywords-Cognitive radio; ad hoc networks; Markov model; queuing theory; tele-traffic capacity; performance analysis;

\section{INTRODUCTION}

Compared to traditional ad hoc networks, Cognitive Radio aided Ad Hoc Networks (CRAHNs) are capable of increasing the achievable capacity by sensing and exploring the white holes in the licensed spectrum in opportunistic ways. However, it remains a challenge to cope with the dynamic characteristics of CRAHNs, including their distributed architecture, multi-hop routing, node mobility and their dynamic 'frequency-hopping' across spectral bands subject to substantial spatio-temporal variance [1-2]. The initial capacity analysis of ad hoc networks was provided in [3], which stimulated numerous subsequent studies including current research on the capacity of CRAHNs, mainly focusing on their theoretical bounds, where the nodes relying on diverse transceivers are typically assumed to be randomly distributed according to various geographic models [4-5]. As a further advance, the authors of [7-8] investigated the practical performance of SUs by setting up a stochastic model of the channel state variations. In [7], the attainable performance was characterized with the aid of the so-called channel occupancy model in which the transition probabilities between channel states were known, but the channel states were only partially observable. The authors of [8] studied a MAC scheme relying on distributed spectrum sensing and the
SUs' performance was characterized by modeling the channel's availability using a two-state Markov chain.

The dynamic variation of the traffic of PUs and SUs is considered by the models in [10-11]. However, these contributions neglected the un-licensed spectrum can only be used by SUs. Although the un-licensed spectrum is unshared, it has a significant impact on sharing of the licensed spectrum. The authors of [12] evaluated the performance of SUs which had access to both the PCs and SCs by. However, their proposed 3-D Markov model is readily not extendable to a large user population. Instead, three queuing models, namely Q1: PCs occupied by PUs, Q2: PCs occupied by SUs, and Q3: SCs occupied by SUs, were proposed to evaluate the performance of SUs. Naturally, these queues influence each other, but these influences are neglected and hence only an approximate evaluation was provided in [12].

This paper provides the capacity and performance analysis of the above-mentioned spectrum sharing model, where the shared PCs are complemented by unshared SCs. We propose two different dynamic spectrum sharing models for analysing the attainable performance of CRAHNs. The first one is a Markov-chain model, which is computationally more complex, but more accurate, while the other is a queue-based model, which is computationally less complex but less accurate. As for the proposed Markov model, our contribution is three-fold. First of all, our Markov-chain model is extendable to an arbitrary practical number of PCs as well as SCs and hence it may be used for the system's accurate performance characterization. Secondly, we demonstrate that our analysis is applicable, regardless of the tele-traffic intensity considered. Thirdly, we also derive a closed-form expression for the maximum NSUPS, which is verified by simulations in order to characterize the capacity upper-bound of SUs operating in practical traffic scenarios. Our further contribution in terms of the proposed queue-based model is also three-fold. Firstly, against the above-mentioned background, we characterize the effects of the coupled relationships between the PCs occupied by SUs and SCs occupied by SUs, so as to improve the modeling accuracy of [12]. Secondly, we will demonstrate that we can reduce the number of queues from three as in [12] to two, in order to simplify the analysis. Finally, we will demonstrate that provided the call arrival rate of PUs is lower

This work has been supported in part by the EPSRC, UK under the auspices of the China-UK Science Bridge and by the RC-UK under the India-UK Advanced Technology Centre (IU-ATC), as well as the National Natural Science Foundation of China (No.61071068, No.60672059), the China Scholarship Council (CSC), the National High-Tech R\&D Program of China (No.2006AA01Z233). 
than that of SUs, the proposed queuing model is extremely accurate in comparison to [12].

The remainder of the paper is structured as follows. In Section II, we described our system, while its basic Markov model and performance analysis are detailed in Section III. In Section IV, we then present an improved queuing model and its performance analysis. Our numerical results are discussed in Section V, while in Section VI, we provide our conclusions.

\section{SYSTEM MODEL}

For simplicity, we assume that all the CR nodes in CRAHNs are statistically speaking identical and independent. For a certain node under consideration, let the Random Variable $(\mathrm{RV}) N_{1}(t)$ represent the number of PCs occupied by PUs and let $N_{2}(t)$ be the sum of SCs and PCs occupied by SUs at time instant $t$, respectively. The following assumptions are made:

1) The maximum number of PCs and SCs within the transmission range of a given node is assumed to be $c_{1}$ and $c_{2}$, respectively. Both of the PUs and the SUs occupy only a single channel per session.

2) The call arrival process of PUs is assumed to be Poissonion with a call-arrival rate of $\lambda_{1}$, while the call holding time of PUs is assumed to be negative exponential associated with a mean value of $1 / \mu_{1}$.

3) The call arrival process of SUs is also assumed to be Poissonion with a call-arrival rate of $\lambda_{2}$, whose call holding time is also assumed to be negative exponential, with a mean value of $1 / \mu_{2}$.

4) When a PU appears in the channel occupied by an active SU, the SU has to sense this event and has to switch to another free PC or to a SC. If there is no other free channel, the SU will be dropped.

5) When a new SU wants to initiate a session, it will be blocked, if all the PCs and SCs are occupied.

\section{MARKOV MODEL AND PERFORMANCE ANALYSIS}

The state space constructed by the vector elements hosting the $\operatorname{RVs}\left\{N_{1}(t), N_{2}(t)\right\}$ may be modeled by a Markov chain having continuous-time parameters. Figure 1 portrays the corresponding State Transition Diagram (STD) for $c_{1}=c_{2}=3$.

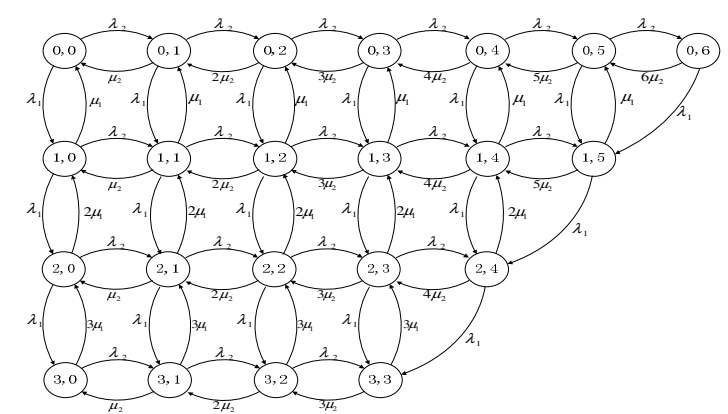

Fig.1. State Transition Graph for Markov model.

For the sake of evaluating the performance of CRAHNs for an arbitrary number of PCs or SCs, the equilibrium equations must be extendable. First, we consider the general relationships seen in the STD of Fig.1. If there are idle channels available at the initial state of the Markov chain, one of the idle channels in either the PC set or in the SC set can be occupied by a new PU or SU. Then we have:

$$
\left(i \mu_{1}+\lambda_{1}+j \mu_{2}+\lambda_{2}\right) p_{i, j}=\lambda_{1} p_{i-1, j}+(i+1) \mu_{1} p_{i+1, j}+\lambda_{2} p_{i, j-1}+(j+1) \mu_{2} p_{i, j+1},
$$

where $i=0, \cdots \cdots, c_{1} ; j=0, \cdots \cdots, c_{1}+c_{2}-i-1$.

By contrast, if no idle channels are available at the initial state of the Markov chain, but a PU intends to initiate a new session, and some PCs are occupied by SUs, then the PU will be given immediate access to a PC occupied by a SU. The interrupted SU session will then be dropped, if no idle SC is available. This scenario is characterized as:

$$
\left(i \mu_{1}+\lambda_{1}+j \mu_{2}\right) p_{i, j}=\lambda_{1} p_{i-1, j}+\lambda_{2} p_{i, j-1}+\lambda_{1} p_{i-1, j+1},
$$

where $i=0, \cdots \cdots, c_{1} ; j=c_{1}+c_{2}-i$.

Then, we can combine eq. (1) and (2) with the probability normalization condition of:

$$
\sum_{i=0}^{c_{1}} \sum_{j=0}^{c_{1}+c_{2}-i} p_{i, j}=1
$$

The corresponding Steady State Probability (SSP) may then be derived by solving the set of equations eq. (1), (2) and (3). Based on the knowledge of the SSP, we can then evaluate the performance metrics of Call Blocking Probability (CBP), Call Dropping Probability (CDP) and NSUPS that may be supported with the aid of the following performance analysis.

\section{A. Performance Analysis}

To start with, let us consider the SU's CBP. A new SU will be blocked if and only if all PCs and SCs are occupied. The CBP for the SUs may be formulated as:

$$
p_{\text {block }}=\sum_{i=0}^{c_{1}} p_{i, c_{1}+c_{2}-i} \text {. }
$$

Let us now consider the SU's CDP. For an active SU, when a new PU arrives, and all PCs as well as SCs are occupied, and assuming furthermore that at least one PC is occupied by a SU, then the PU will opt for occupying the PC currently engaged by the active SU. Unfortunately, the interrupted active SU has no opportunity to switch to another free channel, hence it will be dropped. Then the CDP for each active SU is:

$$
p_{\text {each_drop }}=\sum_{i=0}^{c_{1}-1} p_{i, c_{1}+c_{2}-i} \text {. }
$$

The CDP for SUs is given by the fraction of the active SUs being dropped from the total number of SUs being admitted per unit time interval, namely as:

$$
p_{\text {drop }}=\frac{\lambda_{1} p_{\text {each_drop }}}{\lambda_{2}\left(1-p_{\text {block }}\right)} .
$$

Finally, the normalized NSUPS supported without being dropped and blocked is then given by the average call completion probability of a SU, namely by:

$$
R_{\text {normalized }}^{\text {su }}=\left(1-p_{\text {block }}\right)\left(1-p_{\text {drop }}\right) \text {. }
$$

The actual NSUPS is then given by:

$$
R^{s u}=\lambda_{2} R_{\text {nomalized }}^{\text {su }}=\lambda_{2}\left(1-p_{\text {block }}\right)\left(1-p_{\text {drop }}\right) \text {. }
$$

So far, we have evaluated the performance of SUs by setting up an extendable Markov model capable of handling an arbitrary number of PUs and SUs. The associated computational complexity of solving the equilibrium equations is generally on the order of $\mathrm{O}\left(n^{3}\right)$, where $n$ is the number of states in the Markov chain given by:

$$
n=c_{2}\left(c_{1}+1\right)+\left(c_{1}+1\right)\left(c_{1}+2\right) / 2 \text {. }
$$


Fortunately, the number of $\mathrm{PCs} c_{1}$ and $\mathrm{SCs} c_{2}$ is not too high in practice.

\section{B. The Maximum NSUPS Supported and Their Tele-Traffic}

It can be readily shown that there is no precondition for the SSP's existence in a Markov model [13], hence the Markov model is applicable to any PU's and SU's traffic intensity, which allows us to estimate the maximum NSUPS that can be supported in practice. In Figure 2, we evaluated the NSUPS supported from eq. (8), which shows that upon increasing the call arrival rate of SUs, the NSUPS also increases due to the increased offered traffic. When the call arrival rate of SUs is low, the number of SUs dropped from the set of SUs admitted remains low due to the low conveyed traffic in the system. Therefore, the fraction of SUs completing their call per unit time interval is high, provided the call arrival rate of SUs is low. However, when the call arrival rate of SUs becomes excessive, the fraction of SUs admitted per unit time interval also increases, naturally also increasing the fraction of SUs dropped per unit time. Needless to say that, the total successfully carried tele-traffic tends to a limited maximum value, which is determined by the system's maximum capacity. Below we explore this issue further.

The probability of the event that $i$ PCs are occupied by PUs, when $\left(c_{1}-i\right)$ PCs are available for SUs, is given by (12) in the section IV. When the arrival rate of the SUs tends to infinity, all the channels available for SUs are busy, hence the maximum NSUPS supported by system is given by:

$$
R_{\text {su }}^{u \text { uper-bound }}=\mu_{2} c_{2}+\sum_{i=0}^{c_{1}-1}\left\{\mu_{2}\left(c_{1}-i\right)\left[\left(\lambda_{1} / \mu_{1}\right)^{i} / i !\right] / \sum_{j=0}^{c_{1}} \frac{\left(\lambda_{1} / \mu_{1}\right)^{j}}{j !}\right\} \text {. }
$$

Next, we investigate the maximum NSUPS supported for the specific parameters of $\lambda_{1}=0.25, \mu_{1}=0.5, \mu_{2}=0.4, c_{1}=3,4,5,6$ and $c_{2}=3$, using simulations in order to verify the accuracy of the closed-form formula (10). When using simulations, we assume that the arrival rate of SUs is high, such as $\lambda_{2}=10^{7}$. Then upon substituting $\lambda_{2}=10^{7}$ into eq. (4), (6) and (8), the estimated maximum NSUPS may be readily determined. Similarly, when using the closed-form formula (10) and substituting the above parameters (except $\lambda_{2}$ ) into (10), the maximum NSUPS was plotted in Figure 2. As expected, the theoretical and simulation-based results confirm each other. For example, the maximum NSUPS becomes 2.2025 for $c_{1}=3, c_{2}=3$ for the simulation-based method, while that for the closed-form method is 2.202533676 .

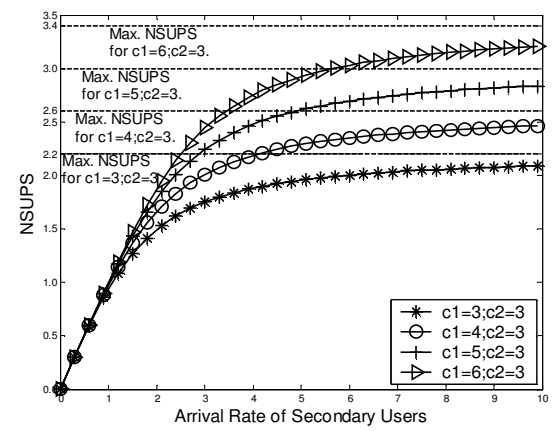

Fig.2. NSUPS supported for $\lambda_{1}=0.25 ; \mu_{1}=0.5 ; \mu_{2}=0.4$. The lines were evaluated from eq. (10), while the symbols by simulations.

\section{QUEUE MODEL AND ANALYSIS}

As mentioned above, in order to reduce the computational complexity of the Markov-chain based method, the authors of [12] set up a queue-based model composed by three subqueues, Q1, Q2, Q3 as mentioned in Section I. This model uses the probability of the state $\left(N^{Q 1}=i ; N^{Q 2}=j ; N^{Q 3}=k\right)$ jointly characterizing the performance, expressed as:

$$
P\left\{N^{Q 1}=i ; N^{Q 2}=j ; N^{Q 3}=k\right\}=P\left\{N^{Q 1}=i\right\} P\left\{N^{Q 2}=j\right\} P\left\{N^{Q 3}=k\right\} .
$$

when they are independent of each other. Naturally, Q1 may be deemed to be a unique independent queue, because the PUs have absolute priority in using the PCs. By contrast, Q2 and Q3 are influenced by Q1, while Q2 and Q3 have an influence on each other, because for example the number of PCs used by SUs influences the number of SCs used by SUs. This interdependence influences the arrival rate or service rate of Q2 and Q3. To elaborate a little further, Q2, is definitely influenced by Q1, since the call dropping or channel swapping events of SUs forced by the PUs in Q1 are expected to increase the service rate of $\mathrm{Q} 2$. Therefore only $P\left\{N^{Q 1}=i\right\}$ is an independent and accurate probability. If we mitigate or eliminate these influences, the accuracy of the joint probability estimate of eq. (11) improves, since (11) was formulated by assuming the independence of the three sub-queues.

Based on the Markov-chain analysis of Section III, we can see from the eq. (1)-(8) that it is the two-component joint probability, but not the three-component one of eq.(11), that is used for obtaining all the results. Hence the performance experienced by the SUs may be characterized with the aid of two, rather than three sub-queues. Below we embark on verifying this hypothesis by evaluating the performance experienced by the SUs relying on a new queue-based model composed by two sub-queues, namely by $N_{1}(t), N_{2}(t)$, as defined in Section II.

Since the PUs enjoy absolute priority in using the PCs, $N_{1}(t)$ may be modeled by a $M / M / c_{1} / c_{1}$ queue with an arrival rate of $\lambda_{1}$ and service rate per channel of $\mu_{1}$ for the PUs. The probability of the event that $i$ PCs are occupied by PUs, which is an accurate probability, is given by:

$$
p_{i}^{(1)}=\frac{\rho_{1}^{i}}{i !} / \sum_{j=0}^{c_{1}} \frac{\rho_{1}^{j}}{j !} .
$$

Assuming that $i$ PCs are occupied by PUs, $N_{2}(t)$ can be modeled by a $M / M /\left(c_{1}+c_{2}-i\right) /\left(c_{1}+c_{2}-i\right)$ queue with a $\mathrm{SU}$ call arrival rate of $\lambda_{2}$ and service rate per channel of $\mu_{2}$. The conditional probability of all channels being occupied under the precondition of $i$ PCs being occupied by PUs, which is an inaccurate probability except for $p_{c_{2}}^{\left(2 l_{1}\right)}$, is formulated as:

$$
p_{c_{1}+c_{2}-i}^{(2 \mid i)}=\frac{\rho_{2}^{c_{1}+c_{2}-i}}{\left(c_{1}+c_{2}-i\right) !} / \sum_{j=0}^{c_{1}+c_{2}-i} \frac{\rho_{2}^{j}}{j !},
$$

where $i=0,1,2, \cdots, c_{1}, \rho_{1}=\lambda_{1} / \mu_{1}, \rho_{2}=\lambda_{2} / \mu_{2}$.

The inaccuracy in (13) arises from the influence imposed by $N_{1}(t)$ on $N_{2}(t)$, which is due to dropping SUs in $N_{2}(t)$ as enforced by the PUs in $N_{1}(t)$, which increases the service rate 
of SUs in $N_{2}(t)$. Note that, only $p_{c_{2}}^{\left(2 \mid c_{1}\right)}$ is accurate in (13), because $N_{1}(t)$ cannot influence $N_{2}(t)$ any more when $i=c_{1}$, i.e. all PCs have been occupied by PUs.

Similar to the Markov-chain analysis of Section III, the CBP for SUs may be formulated as:

$$
p_{\text {block }}=\sum_{i=0}^{c_{1}} p_{i}^{(1)} p_{c_{1}+c_{2}-i}^{(2 \mid i)} \text {. }
$$

The CDP for each active SU is given by:

$$
p_{\text {each_drop }}=\sum_{i=0}^{c_{1}-1} p_{i}^{(1)} p_{c_{1}+c_{2}-i}^{(2 \mid i)} .
$$

The CDP for SUs may be formulated as::

$$
p_{\text {drop }}=\frac{\lambda_{1} p_{\text {each_drop }}}{\lambda_{2}\left(1-p_{\text {block }}\right)} \text {. }
$$

The actual NSUPS supported is given by:

$$
R^{s u}=\lambda_{2} R_{\text {nomalized }}^{\text {su }}=\lambda_{2}\left(1-p_{\text {block }}\right)\left(1-p_{\text {drop }}\right) \text {. }
$$

The joint probability estimate of $p_{i}^{(1)} p_{c_{1}+c_{2}-i}^{(2 \mid i)}$ in eq. (14)-(17) is expected to be more accurate than that in eq. (11). Hence in line with reality, Q2 and Q3 are no longer considered to be independent. As a further benefit, the computational complexity of the joint probability calculation will also be decreased, since it is obtained by multiplying two SSPs, namely those of $N_{1}(t)$ and $N_{2}(t)$ instead of three.

Interestingly, from (14), (15) and (16), we arrive at the relationship between the CBP and the CDP in the form of:

$$
p_{\text {drop }}=\frac{\lambda_{1}\left(p_{\text {block }}-p_{c_{1}}^{(1)} p_{c_{2}}^{\left(2 c_{1}\right)}\right)}{\lambda_{2}\left(1-p_{\text {block }}\right)} \text {, }
$$

where $p_{c_{1}}^{(1)} p_{c_{2}}^{\left(2 \mid c_{1}\right)}$ is an accurate probability according to eq. (12) and (13). Let $\Delta$ be the error of the CBP of SUs, which is formulated as:

$$
p_{\text {block }}^{(*)}=p_{\text {block }}^{(\text {origin })}+\Delta \cdot
$$

The CDP given by the new queuing model is formulated as:

$$
\begin{aligned}
p_{\text {drop }}^{(*)} & =\frac{\lambda_{1}\left(p_{\text {block }}^{(*)}-p_{c_{1}}^{(1)} p_{c_{2}}^{\left(2 c_{1}\right)}\right)}{\lambda_{2}\left(1-p_{\text {block }}^{(*)}\right)}=\frac{\lambda_{1}\left(p_{\text {block }}^{(\text {origin }}-p_{c_{1}}^{(1)} p_{c_{2}}^{\left(2 \mid c_{1}\right)}\right)+\lambda_{1} \Delta}{\lambda_{2}\left(1-p_{\text {block }}^{(\text {origin })}\right)-\lambda_{2} \Delta} \\
& \approx \frac{\lambda_{1}\left(p_{\text {block }}^{(\text {origin) }}-p_{c_{1}}^{(1)} p_{c_{2}}^{\left(2 l_{1}\right)}\right)+\lambda_{1} \Delta}{\lambda_{2}\left(1-p_{\text {block }}^{(\text {orin })}\right)}=p_{\text {drop }}^{\text {(origin })}+\frac{\lambda_{1}}{\lambda_{2}} \cdot \frac{\Delta}{1-p_{\text {block }}^{\text {(origin) }}} .
\end{aligned}
$$

Therefore, when the arrival rate of PUs increases beyond the arrival rate of SUs, the error of CDP will be increased, otherwise, it is extremely accurate, hence the optimum preconditions of its application were explicitly formulated here.

The complexity of evaluating eq. (17) may be shown to be roughly proportional to $\mathrm{O}\left[\left(c_{1}+c_{2}\right)^{2}\right]$, which is significantly lower than that of the Markov-chain model of Section III that is roughly proportional to $\mathrm{O}\left[c_{1}^{6}+c_{1}^{3} c_{2}^{3}\right]$ according to eq. (9).

\section{NUMERICAL RESULTS}

In this section, we characterize the performance experienced by the SUs with the aid of both our Markov-chain model, our queue-based model and the queue-based model of [12]. We investigate how the PUs' arrival rate and the number of PCs influence the performance of SUs for the parameters of $\lambda_{2}=0.2 ; \mu_{1}=0.5 ; \mu_{2}=0.4$ users/second and how the PUs' service rate and the number of PCs affects the performance of SUs for the parameters of $\lambda_{1}=0.25 ; \lambda_{2}=0.2 ; \mu_{2}=0.4$ users $/$ second.

Figure 3 shows that as expected, the CBP increases upon increasing the arrival rate of PUs, because the number of idle PCs decreases upon increasing the traffic load of PUs. In Figure 4, the CBP is shown to decrease upon increasing the service rate of PUs due to having an increased number of idle PCs upon increasing the departure rate of PUs. Figure 5 shows that upon increasing the arrival rates of the PUs, the CDP of SUs increases, because more SUs occupying PCs are forced to terminate their sessions by new PUs. Observe in Figure 6 that when the service rate of PUs tends to 0 , the traffic intensity of PUs tends to infinity. Then there are almost no idle PC for SUs, hence almost no SUs have the opportunity to occupy the PCs. As a result, the CDP of SUs tends to 0 , but this is clearly not a sign of good service for the SUs. When the service rate $\mu_{1}$ of PUs increases, the traffic intensity $\rho_{1}$ of PUs becomes lower, hence there are more idle PCs provided for the SUs, although occupying the PCs is associated with a high risk of being forced to terminate the SU sessions by PUs, when the service rate of PUs is low. Therefore the CDP of SUs increases substantially even for a modest increase of the PUs' service rate, which may actually decrease the holding time of PUs, hence the CDP of SUs begins to decrease when the PUs' service rate increases beyond a certain value. In Figure 7, upon increasing the arrival rate of PUs, the number of PCs available for SUs decreases and thereby the NSUPS decreases. Finally, Figures 3-7 indicate that the CBP and CDP increases, while the NSUPS decreases upon decreasing the number of PCs as well as the number of available PCs.

Comparing the CBP, CDP and NSUPS results of the three models, we can see that the results of Figures 3-7 evaluated from the new queuing model relying on two sub-queues are closer to the ideal result of the Markov model than those of the triple-queue model of [12]. Again, this is because there is only a single inter-dependence between two sub-queues instead of having multiple dependences between three sub-queues, although the match of the CDP and the NSUPS, which is decided by CBP and CDP seen in Figures 5-7 is not as close as that of the CBP seen in Figures 3-4. Eq. (20) provides a physically tangible explanation of this phenomenon and suggests that when the arrival rate of PUs is lower than that of SUs, the proposed queuing model is quite accurate in comparison to that of [12].

We support this conclusion by letting $\lambda_{2}$ vary form 0 to 0.7 for the same other parameters as above. Figure 8 shows that with the increase of the SUs' arrival rate, the number of SUs occupying PCs but being forced to terminate their sessions by PUs increases, hence the CDP also increases. When the arrival rate $\lambda_{2}$ of SUs increases beyond 0.25 , the discrepancy between the results of our new queuing model and the ideal Markovchain model remains small, indicating a better match in comparison to Figure 5 due to the relationship of $\lambda_{1}<\lambda_{2}$. Upon increasing the arrival rate $\lambda_{2}$ of SUs, the probability-estimation error of $\Delta /\left(1-p_{\text {block }}^{\text {(origin })}\right)$ may also increase. Fortunately this errorterm is deweighted by $\lambda_{2}>\lambda_{1}=0.25$ according to eq. (20). 


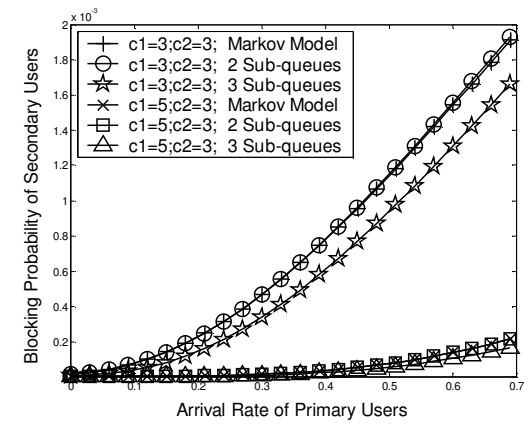

Fig.3. Blocking Probability of SUs vs.the Arrival Rate of PUs

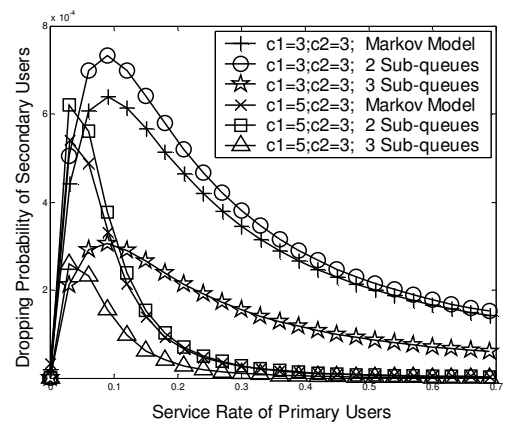

Fig.6. Dropping Probability of SUs vs. the Service Rate of PUs.

\section{CONCLUSIONS}

In this paper, we have proposed two spectrum sharing models considering the SCs as well as the dynamic traffic of PUs and SUs for the performance analysis of CRAHNs. Specifically, a Markov-chain model and a queue-based model was investigated. The proposed Markov model is accurate as well as extendable to an arbitrary practical number of PCs and SCs and to any practical PU and SU traffic intensity. We first pointed out that the Markov model is applicable to any PU and SU traffic intensity, which allows us to assume that the arrival rate of SUs is high, such as $\lambda_{2}=10^{7}$ and thus to determine the maximum NSUPS by a simulation-based method. Furthermore, we have derived the closed-form expression (10) for the maximum NSUPS for SUs operating in practical traffic scenarios. In contrast to the Markov-chain model, the queuebased model has a lower complexity, although at the cost of being more inaccurate. Moreover, our analysis provided by eq. (20) and the simulation results of Figures 5 and 8 indicate that provided the arrival rate of PUs is lower than that of SUs, the accuracy of the CDP of SUs evaluated from the proposed queuing model is high. In a nutshell, our numerical results verify the efficiency and accuracy of the proposed models. In our future research, diverse spectral and transmission models will be studied $[2,6,9]$.

\section{REFERENCES}

[1] J. Mitola and G. Maguire, "Cognitive radio: Making software radios more personal," IEEE Pers. Commun., vol. 6, no. 4, pp. 13-18, Aug. 1999.

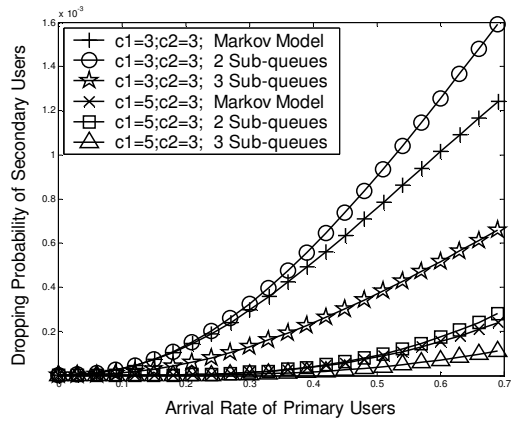

Fig.5. Dropping Probability of SUs vs.the Arrival Rate of PUs

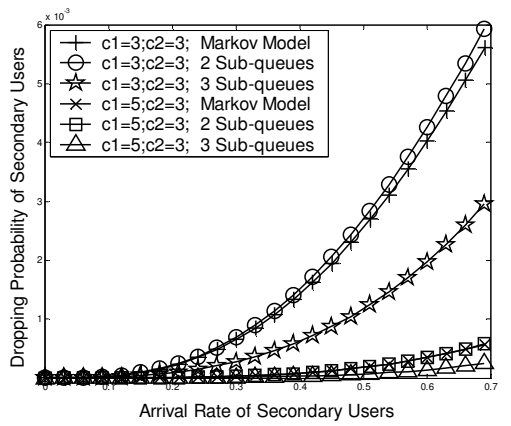

Fig.8. Dropping Probability of SUs vs.the Arrival Rate of SUs

[2] I. F. Akyildiz, W. Lee, M. C. Vuran, and S. Mohanty, "Next generation/dynamic spectrum access/cognitive radio wireless networks: A survey," Elsevier Computer Network, vol. 50, pp. 2127-2159, Sep 2006.

[3] P. Gupta and P. R. Kumar, "The capacity of wireless networks," IEEE Trans. Inf. Theory, vol.46, pp.388-404, Mar. 2000.

[4] C.X. Wang, X. Hong, H.H. Chen, J. Thompson,"On Capacity of Cognitive Radio Networks with Average Interference Power Constraints," IEEE Trans. Wireless Commun., Vol. 8, No. 4, pp. 1620 1625, April 2009.

[5] M. Gastpar. "On Capacity Under Receive and Spatial Spectrum-Sharing Constraints," IEEE Trans. Inf. Theory, Vol. 53, No. 2, pp 471-487, Feb. 2007.

[6] K. B. Letaief and W. Zhang, "Cooperative communications for cognitive radio networks," Proc. IEEE, vol. 97, no. 5, pp. 878-893, May 2009.

[7] Q. Zhao, L. Tong, A. Swami, and Y. Chen, "Decentralized Cognitive MAC for Opportunistic Spectrum Access in Ad Hoc Networks: A POMDP Framework," IEEE J. Sel. Areas Commun., vol. 25, no. 3, pp. 589-600, Apr. 2007.

[8] H. Su and X. Zhang, "Cross-Layer Based Opportunistic MAC Protocols for QoS Provisionings Over Cognitive Radio Wireless Networks," IEEE J. Sel. Areas Commun., vol. 26, no. 1, pp. 118-129, Jan. 2008.

[9] L. Hanzo, O. Alamri, M. El-Hajjar, N. Wu, "Near-Capacity MultiFunctional MIMO Systems: Sphere-Packing, Iterative Detection and Cooperation," Chichester, UK: Wiley, 2009.

[10] X. Zhu, L. Shen, and T. P. Yum, "Analysis of cognitive radio spectrum access with optimal channel reservation," IEEE Commun. Lett.,vol.11, no. 4, pp. 304-306, Apr. 2007.

[11] W. Ahmed, J. Gao, H. A. Suraweera and M. Faulkner, "Comments on "Analysis of Cognitive Radio Spectrum Access with Optimal Channel Reservation"," IEEE Trans. Wireless Commun., vol.8, no.9, pp. 44884491, Sep. 2009.

[12] H. Al-Mahdi, M. A. Kalil, F. Liers, and A. Mitschele-Thiel, "Increasing Spectrum Capacity for Ad Hoc Networks using Cognitive Radios: An Analytical Model," IEEE Commun. Lett.,vol.13, no. 9, pp.676-678, Sep. 2009.

[13] Z. Wang, X. Yang, "Birth and Death Processes and Markov Chains," Springer, 1993. 